\title{
AIRBORNE BATTLE MANAGEMENT SYSTEM \& AUTONOMOUS OPERATIONS UAV AUTONOMY MMIs
}

\author{
Dr. Gerald S. Mersten \\ NAVAIR \\ Patuxent River, MD
}

\begin{abstract}
Both the Airborne Battle Management System (ABMS) Program and the Autonomous Operations (AO) Future Naval Capabilities (FNC) Unmanned Air Vehicles (UAV) Autonomy Program are addressing increased crew multi-tasking and interruptions and are investigating technologies that would enable the Man-Machine Interface (MMI) to support human multi-tasking, help negotiate alerts and interruptions, and promote smooth resumption of interrupted tasks. Our R\&D interests in these areas is "how to enhance crew coordination by creating systems that can anticipate coordination breakdowns / overloads and take action to prevent and/or remedy these situations". These actions could be something as simple as a console alert saying "You don't need to do this; so-and-so (a biological or silicon unit) has already started this task".
\end{abstract}

\section{Background}

\section{UAV Autonomy}

UAV Autonomy is part of the AO FNC. This effort is a new start program, which is currently in the program definition phase.

UAV Autonomy Vision - Enhance the mission capability of Naval Forces by developing technologies that will dramatically increase the performance and affordability of Naval organic UAVs. The overall objective is the development and demonstration of the capability for Naval UAV's to (1) provide access to battlespace areas of responsibility, (2) perform automated surveillance and reconnaissance in all conditions, (3) process surveillance and reconnaissance sensor data, (4) communicate with the $\mathrm{C} 2$ network and other platforms, and (5) minimize the amount of human intervention required to conduct their missions.

UAV Autonomy Goals - Develop an autonomous, intelligent, real-time surveillance and reconnaissance and/or combat unmanned air vehicle (UAV/UCAV) capability:

- To achieve operational capability comparable to manned aircraft for certain types of missions;

- To achieve an affordable high level of autonomous Mission Effectiveness, Safety and Survivability;

- For Distributed, Collaborative and Coordinated Operation with other UAVs/UCAVs and manned A/C;

- For Planining under Uncertainty against an intelligent adversary;

- For Independent Action / Adaptation;

- With Situation \& Self Awareness.

UAV Autonomy Objective/Description Develop critical technologies that address capability gaps for UAVs/UCAVs. These capability gaps include limited human operator intensive operations (command, control, etc.), SA, high bandwidth requirements, limited lost communications capability, limited fault tolerance, limited multivehicle coordination, and limited ability to operate in all types of airspace. The critical technology groups are Situation Awareness (SA), MultiVehicle Networks / Communications / Operations, and Intelligent Autonomy (IA). SA includes technologies for sensor data processing to enable adaptation and independent action with operator alerts and pre-programmed reactions, limited area search using the sensor payload to automatically recognize pre-programmed targets utilizing automatic target cueing / automatic target recognition / combat identification

\section{U.S. Government work not protected by U.S. Copyright.}


(ATC/ATR/CID) techniques, intelligent information management \& dissemination, simple / integrated display of vehicle system status with operator pull for information on demand and includes expanded ship operating envelope. The Multi-Vehicle Networks / Communications / Operations develop / provide communications architecture for networking and multi-vehicle operations, $\mathrm{C} 2$ and information display for multiple simultaneous imaging missions (using a single UAV operator). The Intelligent Autonomy activities develop the ability to make tactical and strategic decisions based on perceptions derived from acquired data. The IA activities will develop intelligent vehicle self-management, intelligent autonomous control (reliable control, autonomous mission management, autonomous battle management, autonomous redundancy management, autonomous communications management, autonomous health / usage \& fault tolerance management, autonomous payload management, autonomous sensor data management, autonomous sensor management, autonomous flight controls, autonomous vehicle management), and reasoning algorithms (significantly beyond the current UAV state of the art), dynamic / autonomous in-flight mission re-planning (including techniques for planning under uncertainty), coordination and control techniques, autonomous threat reaction (and operator alert), robust / cooperative interoperability with manned and unmanned aircraft, "no / lost link" autonomous operations, voice recognition for command and control, and extremely robust ship operating envelope.

Payoffs - Increased UAV (1) access to battlespace areas of responsibility, (2) automated surveillance and reconnaissance in all conditions, (3) automated processing of surveillance and reconnaissance sensor data, (4) communicate with the $\mathrm{C} 2$ network and other platforms, and (5) minimize the amount of human intervention required to conduct their missions while removing the human from the D3 (dull, dirty and dangerous) missions, mission success rate and reliability, reduction in UAV vehicle and payload control manpower, increased situational awareness for commanders and operations centers, expanded ship operating envelope and intelligent UAVs capable of autonomous operations.
Challenges - Technologies must be validated to sufficient levels of maturity (ready for technology transition) to be used to meet the goals and payoffs.

Planned/Expected date of availability and primary contributions - Technology improvement in situation awareness and signal data processing (demonstration in FY03 and transitions beginning in FY04); multi-vehicle operations and communications / networking (demonstration in FY05 and transitions beginning in FY06); and intelligent autonomy (demonstration in FY07 and transitions beginning in FY08) are the planned and expected contributions.

\section{Airborne Battle Management System (ABMS)}

The Navy under the ABMS program, sponsored by ONR Code 31 (Command, Control and Combat Systems) and executed by NAVAIR, is addressing the shifting focus of naval operations to power projection in a littoral environment, as expressed in "Forward from the Sea" and "Operational Maneuver from the Sea". To effectively project naval power into the littoral environment with minimal reliance on a heavy footprint ashore, the force will require extensive NCW support from C4ISR located on the "high ground" provided by naval aviation. ABMS specifically addresses the problem of "How to get the right information / image(s) to the right pilot(s)/ platform(s) soon enough?" in order to provide Navy and Marine Corps aviation the ability to observe, direct, and control from high above the littoral battlespace. This will be central to our ability to respond quickly and accurately to threats deep inland and to employ ship-launched, air-launched, and ground-launched weapons with maximum effect. Without a heavy naval footprint, the need for MMIs to reduce the workload increases in importance.

ABMS provides substantial reduction in the engagement timeline for time critical and/or mobile targets through the development of and/or the integration of critical sensor-to-shooter technologies. Specifically, ABMS addresses Continuous Surveillance/Battle Damage Assessment, Sensor Management, Information 
Gathering \& Management, Information

Dissemination, Target/Combat ID, Multi-mission

Coordination and De-confliction, Dynamic Rules of

Engagement / Air Tasking Order (ROE / ATO) and

other guidance, Targeting, Target / Weapon Pairing

Rate, Flight Path Routing and Threat Avoidance,

Precision Fire, Automated Distributed /

Decentralized Weaponeering (C2), and Platform

Survivability.

ABMS specifically addresses the problem of "How to get the right information / image(s) to the right pilot(s)/platform(s) soon enough?" by shortening the "Decide" phase of both the Detect, Decide, Engage and BDI/BDA Assessment aspects of the kill chain and the OODA (Observe, Orient, Decide, Act) cycle. ABMS is three fold in that it is (1) developing and demonstrating the implementation concepts for intelligent in-flight 4$D$ (space - time) image management and dissemination, namely the "Image Editor" (Surveying/Anomaly Detection, Chicleting, IDing, \& Filtering), "Router" (Selecting, Geo-Sorting, \& Disseminating), and "Sorter" (Time-

Sorting/Posting, Geo-Registering to 3-D Terrain Database, and Displaying when Relevant) for bandwidth / timeline reduction, (2) developing and demonstrating ATC and CID to recognize 3-D objects (discriminating between targets and friendlies / neutrals) and discriminating between 3D objects and 2-D decoys, and (3) developing and demonstrating the "plug \& play" system integration of a tactical airborne Network Centric information / image battle management and $\mathrm{C} 2$ architecture (which includes dynamic ROE, ATO, etc.), georegistration software tools, various image processing software applications and ABMS' Image Editor/Router/Sorter.

Naval aviation represents a unique resource beyond the evident use for sensing and strike. This is an element of the forward force with minimal distance to the enemy, minimal response time to threats (both offensively and defensively); location on a "virtual high ground" that can be as high as Mount Everest, and potential proximity to the enemy that is matched only by ground forces in close combat. Operational commanders have strived for centuries to capture the "high ground" as a location from which to observe, command, and control the battle, and naval aviation offers a significant potential to establish this advantage for our forces.

These advantages are offset by obvious limitations in the ability to support warfighting functions in aircraft that can operate from bases afloat. Limitations include factors such as saturated workload for the pilots and small airborne staffs as well as coordination issues between crew members / tasks; limited bandwidth, processing, memory, and display resolution/definition; increased tempo / rate of change of the battlespace when conflict breaks out; and determining / providing functionally tailored information to the warfighters (what the warfighter needs) when they need it. Other issues include obtaining and integrating off-board and onboard information / images, the need for immediately comprehendible information, "timelate" information, skin-to-skin architectural implications, etc., real-time node characteristics within the network-centric architecture for an embedded information management system in the platform to manage sharing information (e.g., graphics \& imagery, air / ground threats, re-tasking / re-planning) with other forces to support a realtime all-source information / image based sensor-toC2-to-shooter picture of the battlespace, and affordability and retrofit issues (including shortfalls and architectural limitations of legacy aircraft). There is also the need to incorporate the architecture and infrastructure to produce an enterprise wide low latency tactical Single Integrated Battlespace Picture (SIBP). Another major issue is the absence of an integrated "plug \& play" tactical airborne Network Centric information / images battle management and $\mathrm{C} 2$ architecture for the above critical sensor-to-shooter technologies.

\section{Technical Concept}

Our overall concept combines the extensive use of real-time, man-in-the-loop modeling and simulation tools with end-user task analysis and observational data collection. Our use of modeling and simulation tools early in the program facilitate the technology base development and evaluate system performance and will include the use of mission models to assess performance in a wide range of operating environments. Applying experience in conducting user needs assessments and analyzing observational data will allow us to 
effectively include the war-fighter in these early experiments to set priorities and establish preliminary operating procedures and interactions. We envision demonstrations in these simulation environments to be key milestone events to invoke operator inputs and mitigate technology development risk. As exit criteria is met in these early stages, system functions need to be incorporated into tactical exercises such as the FNC demonstrations and Naval Warfare Development Center's Fleet Battle Experiments.

This concept addresses a second need, for network-centric mission coordination among $\mathrm{C} 2$, sensor, and strike assets without overloading the crews whether they be the air crews in the manned $\mathrm{C} 2$, sensor and strike assets or the ground crews "operating" the UAVs/UCAVs. Time critical operations, limited bandwidth, and increased crew workload environments conspire against conventional solutions. However, industry has developed innovative solutions to distribute computing for battlefield operations using mobile agents to encapsulate, migrate, and share mission critical information. This technical approach adopts agent-enhanced information networks that enable such capabilities as airborne route-planning systems to maintain coordination with each other and with ground or airborne-based C2 centers while facilitating enhanced MMI.

System technologies and interface design concepts are designed through a spiral development process. This process will use standard system engineering practices to identify system requirements and to push technology advances into system capabilities. A typical program approach includes 1) the development of concepts of operations for integration into military operations, 2) the definition of system functions, 3) the identification of candidate system solutions and architectures, and 4) the experimentation and demonstration to provide the framework for concept of operations development and transitions into the fleet.

\section{Technical Approach}

Our transition approach is centered on two primary goals: 1) rapid conversion of newly developed capabilities and algorithms into affordable products and 2) creation of a robust, field-capable design targeted for broad transition and reuse across multiple weapon system platforms. These goals will be achieved through exploitation of widely available commercial based tooling, standards, and support software as well as the development of other needed capabilities. In addition, our transition approach employs a lean capability validation process along with modular production design practices that have been systematically matured over the past decade in combat-fielded systems. Collectively, the features of our approach have been shown effective in achieving affordable transition of new capability applications into large-scale DoD embedded systems.

This transition plan leverages a proven method to transition algorithms and technologies into production system platforms and host processors as depicted in Figure 1 Development Environment Process, and Figure 2 Software Production Method. We have experience in maturing avionics and pilot vehicle interface system concepts by testing and evaluating prototype algorithms and code in rapid algorithm prototype stations (RAPS). Once the stability of the algorithm and software is demonstrated, the solution is transitioned to a virtual simulation environment that further validates the system concept and matures the interoperability of system performance in an existing or emulated environment. This approach uses our SEI Level 4 certified software development process and integral testing and evaluation environments to efficiently transition capabilities into embedded processors. Following initial laboratory experimentation where necessary, selected field exercises will focus on demonstration of the technology to prospective users. It is at these system level demonstrations where military utility of the technologies will be fully assessed and transition potential demonstrated. This development cycle is designed to minimize technology and program risk through a spiral evaluation process. 


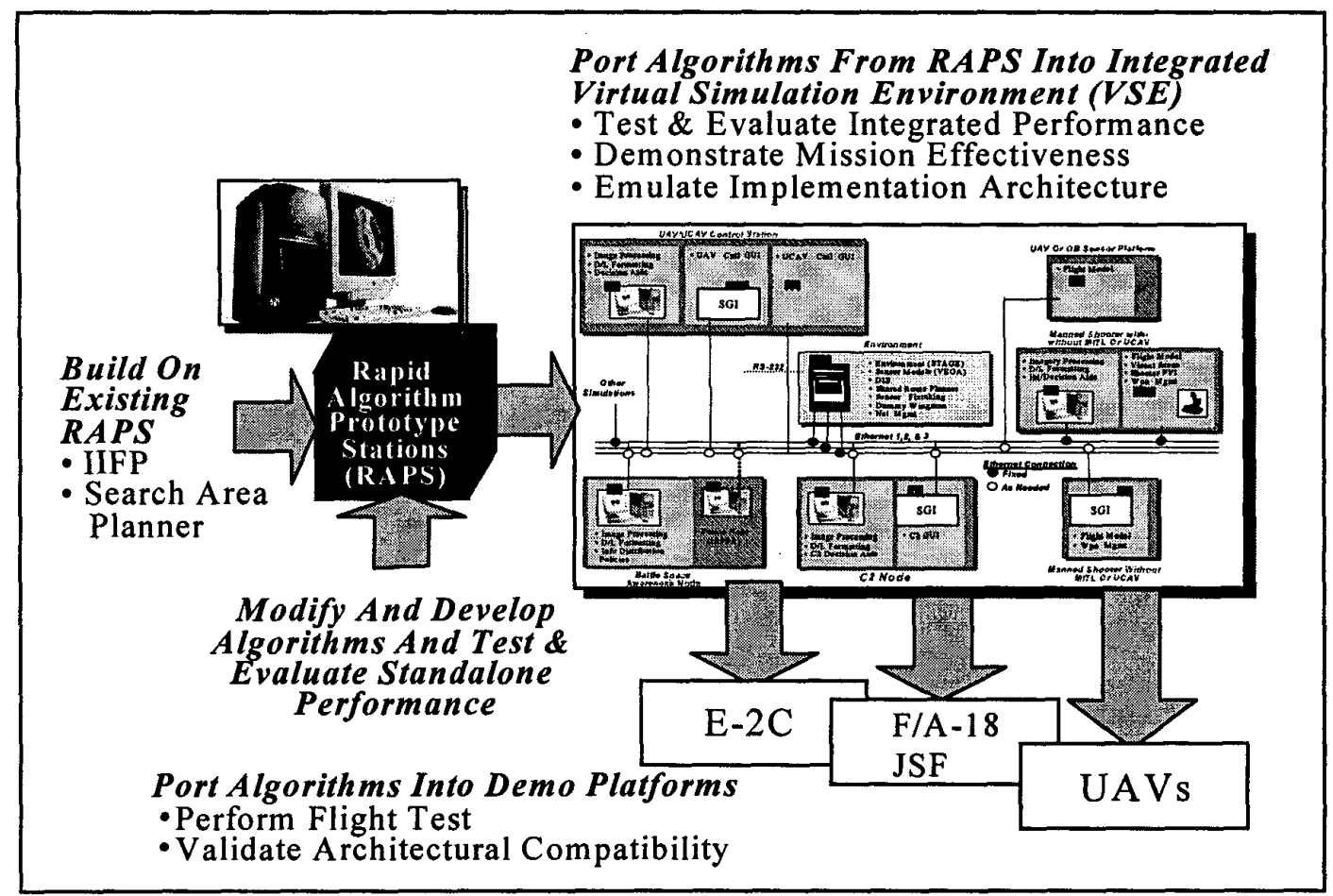

Figure 1 Development Environment Process (Using ABMS as Example)

Mainstream COTS development tools, operating systems, languages and middleware are key to affordable production development as they allow the $\mathrm{DoD}$ to leverage large commercial economies of scale.

\section{ABMS}

The ABMS program utilizes a System Engineering \& Integration (SE\&I) team consisting of Lockheed Martin (lead) and Draper Laboratory. LM development teams use broadly employed standards such as POSIX, Unified Modeling Language (UML), $\mathrm{C}+$, and CORBA, which allow newly fielded capabilities to be easily ported across development environments and to multiple target hardware platforms. In addition, LM-ATL is a leading developer of mobile agent systems compatible with DARPA's CoABS Grid, an emerging standard in interoperable, network centric systems and in MMI systems. Likewise, Draper provides complementary and compatible capabilities. This combination helps eliminate customized or "stovepipe" designs and resists diminishing manufacturing sources (DMS) effects, keeping new capabilities viable across long service life cycles. Commercial automation capabilities are also fully leveraged during software development. Our production approach exploits automatic generation of application source code from modelbased representations and utilizes a variety of testing automation services.

Our team utilizes common software architectures and employ lean modular design methods based on Object Orientation (OO). Key features include the ability to modularize complex applications, rigorous control of software component interfaces, and exploitation of object "classes" in capturing reusable application content. Our development teams also have extensive experience developing application adapter modules and "wrappers". This capability allows newly developed software to be easily injected into legacy designs that may not meet modern commercial standards. 


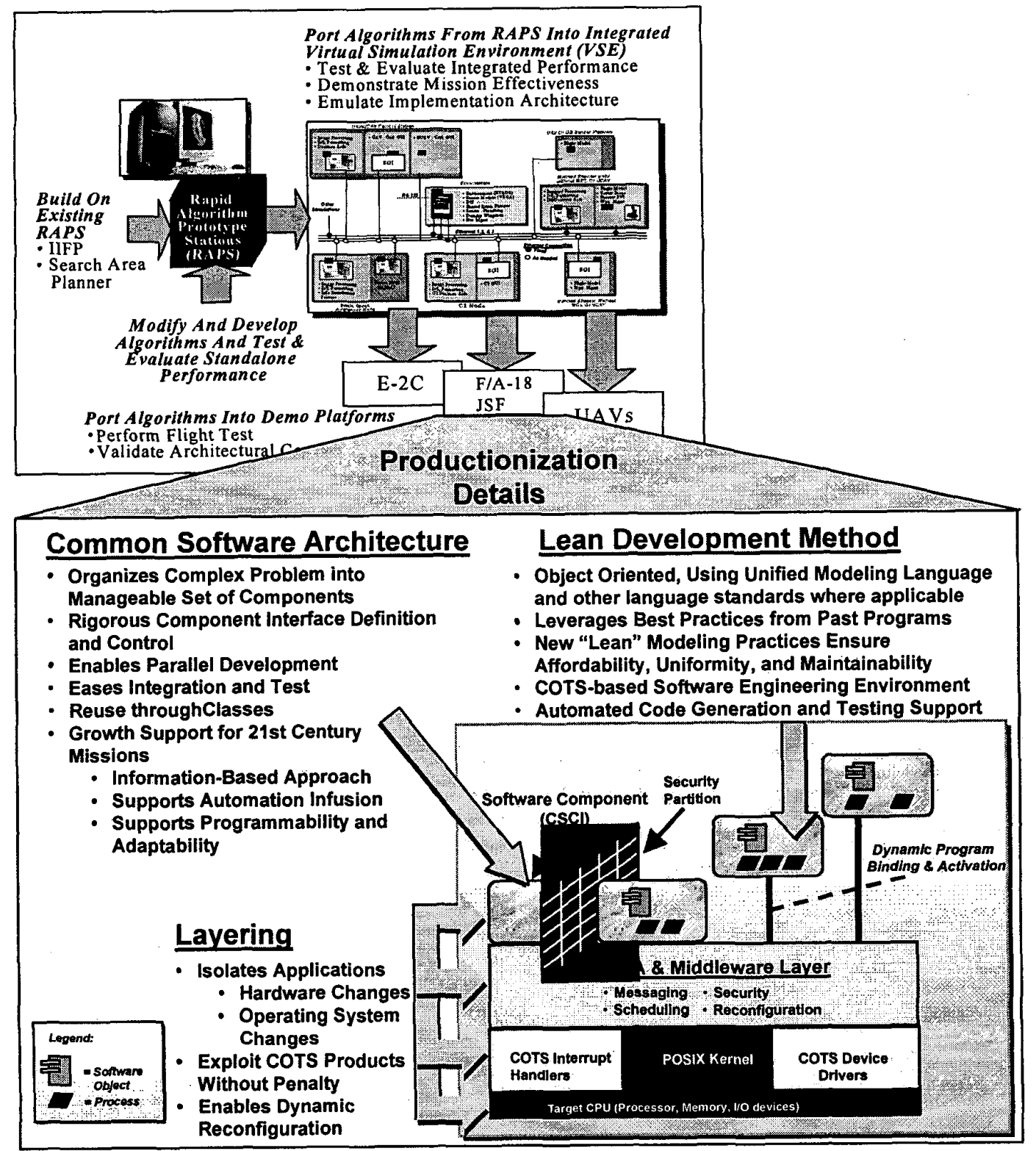

Figure 2 Software Production Method (Using ABMS as Example) 


\section{UAVAutonomy}

The UAV Autonomy Program in FY01 is in the definition phase and has several study contractors (including Draper Laboratory, Northrop Grumman, Lockheed Martin and Technology Strategic Planning) performing various support efforts related to SA, Multi-vehicle Networking / Communications / Operations, and Intelligent Autonomy. In FY02 - FY07 the program will be executed around these main interest areas. The current requirements that the team is working to address (although subject to change) include:

a. Automatic detection of (1) threats (threshold: anti-aircraft artillery airbursts [flak], rising tracer rounds, missile launches with smoke trail; objective: threshold + missile launch without smoke, threat aircraft), (2) collision situations (threshold: IFF equipped aircraft; objective: birds, nonIFF equipped aircraft, weather, towers), (3) terrain (threshold; rising terrain along flight path identified in database; objective: rising terrain within five minutes flight time [not in database]), (4) targets of opportunity (threshold: heavy military equipment [armored vehicles, artillery, aircraft] in the open; objective: heavy military equipment [armored vehicles, artillery, aircraft] in the open or partially concealed)\} with an overall threshold of an operator alert within five seconds, pre-programmed aircraft or sensor maneuver initiation easily accessible to the UAV operator (if action required) while using sensors capable of being mounted on the UAV and an overall objective of an operator alert within one second, preprogrammed aircraft or sensor maneuver initiated autonomously (if action required) while using only baseline sensors),

b. Automatic recognition (threshold: recognition as to class (e.g. armored vehicle, aircraft, etc.) when target is in the open; objective: identification as to type (e.g. T-72 tank, MIG-29 aircraft, etc.) when in the open or partially concealed),

c. Vehicle system status consisting of (1) single icon display (threshold: single icon display of rolled-up vehicle health and status information; objective: threshold + trend prediction. User friendly display of key state change information and recommended action [if required]) and (2) operator pull for information on demand (threshold: ability for UAV operators to access detailed vehicle information on demand [in user and mission friendly way]; objective: same as threshold),

d. Multi-vehicle networking / communications / operations (threshold: single, aviation rated operator commands multiple UAVs; objective: single non-aviation rated operator commands multiple UAVs).

\section{Summary}

As mission crews (both airborne crews as well as UAV "operators") accept greater responsibilities for either an integrated airborne battle management function or operation of UAVs, the potential arises for workload-induced performance degradations. Operators must be able to complete ABMS and UAV-enabled missions in the face of increased multi-tasking and interruptions. To ensure the success of both the ABMS and UAV Autonomy programs, we are investigating technologies that would enable the Man-Machine Interface to support human multi-tasking, help negotiate alerts and interruptions, and promote smooth resumption of interrupted tasks. A theoretical framework called Human Alerting and Interruption Logistics (HAIL) is one approach to supporting human multi-tasking (McFarlane, 1999). HAIL assists users by negotiating the manner in which tasks are interrupted and resumed, applying findings from empirical studies of subjects who are interrupted in various ways while performing a sequence of tasks. HAIL (funded in part to date by the Office of Naval Research's ABMS program and by LM-ATL) will now transition from concept to technology under both the Office of Naval Research's Decision Support Systems Future Naval Capabilities and ABMS) so that HAIL technologies can be incrementally incorporated into various Naval systems.

\section{References}

McFarlane, D.C. (1999). Coordinating the Interruption of People in Human-Computer Interaction. In Proc. of Human-Computer Interaction - INTERACT'99. IOS Press, Inc., The Netherlands, IFIP TC.13, 295-303. 